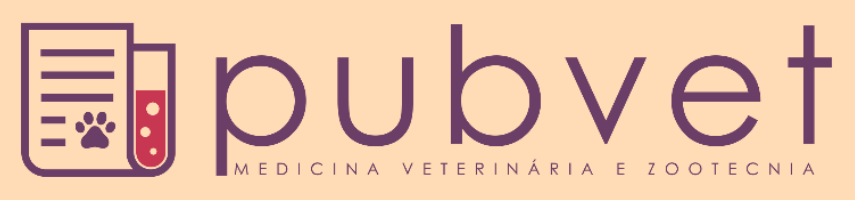

https://doi.org/10.31533/pubvet.v14n5a576.1-8

\title{
Determinação do parto em cadelas através da mensuração ultrassonográfica de estruturas fetais e extrafetais
}

\author{
Maíra Planzo Fernandes*1 ${ }^{\bullet}$ (D), Marcus Vinícius Galvão Loiola ${ }^{\bullet}$, Antonio de Lisboa Ribeiro

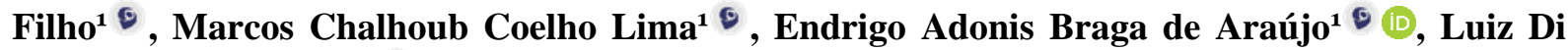 \\ Paolo Maggitti Junior ${ }^{1} \odot$ \\ ${ }^{1}$ Universidade Federal da Bahia, Escola de Medicina Veterinária e Zootecnia, Setor de Reprodução Animal e Obstetrícia \\ Veterinária, Salvador, BA, Brasil \\ *Autor para correspondência: maira_planzo@yahoo.com.br
}

\begin{abstract}
Resumo. A presente revisão de literatura tem como objetivo abordar o uso da ultrassonografia como método de diagnóstico gestacional em cadelas e, sua utilização no acompanhamento do desenvolvimento e viabilidade fetal, na determinação da idade gestacional e data provável do parto em cadelas. O tempo de gestação canino é relativamente curto, levando ao desenvolvimento dos principais órgãos fetais no seu período final, e ao nascimento de fetos imaturos. A determinação da idade gestacional permite estimar a hora do parto, de modo que criadores e veterinários possam ajudar a reduzir as perdas do periparto. A medição ultra-sonográfica de estruturas extra-fetais e fetais é um método comum e preciso para a previsão da data de parto durante a gestação canina, no qual serão escolhidas a depender do período gestacional da fêmea. O diâmetro da cavidade coriônica interna (ICC) é considerado o mais preciso indicador de idade gestacional na cadela entre os dias $20^{\circ}$ e $37^{\circ}$, já o diâmetro biparietal (DBP) é um parâmetro confiável no terço final da gestação, entre os dias 38 e 60 . As variações raciais em cadelas podem interferir nos cálculos da idade gestacional a partir de medidas fetais, sendo necessário estabelecer padrões de medidas e cálculos para animais de um mesmo porte ou característica racial.
\end{abstract}

Palavras chave: canino, biometria fetal, idade gestacional

\section{Determination of delivery in bitches through ultrasound measurement of fetal and extrafetal structures}

\begin{abstract}
Abastract. The aim of this study was to evaluate the use of ultrasound as a method of gestational diagnosis in female dogs, and its use in monitoring fetal development and viability, in determining gestational age and probable date of delivery in female dogs. The canine gestation time is relatively short, resulting in the development of the main fetal organs in their final period, and birth of immature fetuses. Determining gestational age allows the time of birth to be estimated, so that breeders and veterinarians can help reduce peripartum loss. Ultrasound measurement of extra-fetal and fetal structures is a common and accurate method for predicting the date of delivery during canine pregnancy, in which they will be chosen depending on the gestational period of the female. The inner chorionic cavity diameter (ICC) is considered the most accurate indicator of gestational age between the 20th and 37th days, whereas biparietal diameter (BPD) is a reliable parameter in the final third of pregnancy, between the 38th and 60th days. Breed variations in dogs may interfere in gestational age calculations based on fetal measurements, and it is necessary to
\end{abstract}


establish measurement standards and calculations for animals of the same size or breed characteristics.

Keywords: canine, fetometry, gestation age

\section{Determinación del parto en perras a través de la medición por ultrasonido de estructuras fetales y extrafetales}

Resumen. La presente revisión de literatura tiene como objetivo abordar el uso de la ecografía como método de diagnóstico gestacional en perras y su uso en el monitoreo del desarrollo y la viabilidad fetales, para determinar la edad gestacional y la fecha probable de parto en perras. El tiempo de gestación canina es relativamente corto, lo que lleva al desarrollo de los principales órganos fetales en su período final y al nacimiento de fetos inmaduros. La determinación de la edad gestacional permite estimar el momento del parto, de modo que los criadores y los veterinarios pueden ayudar a reducir las pérdidas periparto. La medición por ultrasonido de las estructuras extra fetales y fetales es un método común y preciso para predecir la fecha de parto durante la gestación canina, que se elegirá según el período de gestación de la hembra. El diámetro de la cavidad coriónica interna (ICC) se considera el indicador más preciso de la edad gestacional en las hembras caninas entre los días $20^{\circ}$ y $37^{\circ}$, mientras que el diámetro biparietal (DBP) es un parámetro confiable en el tercio final de la gestación, entre los días 38 y 60. Las variaciones raciales en las perras pueden interferir con los cálculos de la edad gestacional basados en mediciones fetales, por lo que es necesario establecer estándares de medición y cálculo para animales del mismo tamaño o características raciales.

Palabras clave: canina, biometría fetal, edad gestacional

\section{Introdução}

A gestação canina é um evento singular e particular entre as demais espécies de mamíferos. O tempo de gestação da cadela é relativamente curto, levando ao desenvolvimento dos principais órgãos fetais no seu período final, com nascimento de fetos imaturos (Lopate, 2018). Aliado a isso, os tutores de cães vêm se tornando cada vez mais exigentes na procura de informações sobre a saúde dos seus animais. Atualmente, proprietários e criadores buscam informações relacionadas à confirmação de prenhez, desenvolvimento e viabilidade fetal e contagem do número de fetos (Baba, 2010).

A determinação da idade gestacional permite estimar a hora do parto, de modo que criadores e veterinários possam planejar um parto assistido, se necessário, ajudando, assim a reduzir as perdas do periparto (Kutzler et al., 2003). Diante do exposto, o exame ultrassonográfico tem sido utilizado no exercício da Medicina Veterinária tanto para detectar precocemente a gestação como para o estudo do desenvolvimento fetal (Zambelli \& Prati, 2006), além de, por meio do seu uso, a idade gestacional poder ser avaliada (Giannico et al., 2016). A medição ultra-sonográfica de estruturas extra-fetais e fetais é um método comum e preciso para a previsão da data de parto durante a gestação canina, mesmo quando o tempo de ovulação é desconhecido (Beccaglia et al., 2016).

Essas estruturas podem ser mensuradas de acordo com o dia de gestação da cadela. O diâmetro interno da cavidade coriônica é o mais comumente usado para prever o dia do parto no início da gravidez, enquanto que o diâmetro biparietal é amplamente aplicado no segundo semestre de gravidez (Luvoni \& Beccaglia, 2006). Sendo assim, a presente revisão de literatura tem como objetivo abordar o uso da ultrassonografia como método de diagnóstico gestacional em cadelas, enfocando sua utilização no acompanhamento do desenvolvimento e viabilidade fetal, na determinação da idade gestacional e data provável do parto em cadelas.

\section{Fisiologia da gestação canina}

Nas cadelas a ovulação ocorre 24 a 96 horas após o pico do Hormônio Luteinizante (LH). Neste período, os oócitos ainda não estão fertilizáveis, pois são ovulados como oócitos primários, sem a liberação do primeiro corpúsculo polar, e sofrerão a maturação em 2 a 4 dias nas tubas uterinas 
(Concannon et al., 2001; Concannon et al., 2003). Após esse período, pode-se ter a fecundação, união do gameta masculino com o gameta feminino, o qual ocorre no segmento distal da tuba uterina, região conhecida como istmo (Tsutsui, 1989).

O espermatozoide canino pode resistir e permanecer fértil por pelo menos 4 a 6 dias no sistema genital da fêmea. Portanto, a concepção pode ocorrer em até 7 dias após o último acasalamento. (Baba, 2010). Tal fato, aliado a liberação de oócito imaturo e a capacidade da cadela aceitar a monta várias vezes, resulta na dificuldade em se estabelecer, com precisão, o tempo gestacional nessa espécie (Leite, 2003). Os oócitos fertilizados são mantidos dentro das tubas uterinas por 9 a 10 dias e entram no útero no estágio de mórula (Songsasen \& Wildt, 2007). Esta mórula é constituída por uma camada interna, o qual dará origem aos tecidos do embrião propriamente dito e a camada externa, responsável por dar origem ao trofoblasto e, contribuir na formação da placenta (Ferrari, 2009). Por fim, ocorre a implantação, no qual se dá 18 a 21 dias após a ovulação (Reynaud et al., 2006).

Em relação aos hormônios gestacionais, para o desenvolvimento da gestação, há necessidade de altas concentrações de progesterona (P4), que na cadela é produzida exclusivamente pelos corpos lúteos (CLs) (Luz et al., 2005). A secreção lútea de progesterona depende tanto do LH hipofisário quanto da prolactina (Concannon et al., 1989, 2003) que, por sua vez, durante o segundo período de gravidez canina, é a principal agente luteotrófico (Johnson, 2008).

Com a maturação fetal ao final da gestação, o aumento do hormônio adrenocorticotrófico (ACTH) leva à subsequente liberação de cortisol fetal. Esse aumento do cortisol terá como consequência o declínio abrupto de P4, aumento de estrógenos e, liberação de prostaglandina F2 alfa (Luz et al., 2005). Tais alterações hormonais serão as responsáveis por levar ao desencadeamento do parto.

\section{Duração da gestação canina}

A idade gestacional canina pode variar de acordo com o método utilizado para determinar seu início (Camargo, 2012). Assim, pode-se considerar, como tempo de gestação nessa espécie, o intervalo de 63 \pm 7 dias se for calculado a partir da data da primeira cobertura, $65 \pm 1$ dia se for calculado a partir do pico de $\mathrm{LH}$, e $57 \pm 3$ dias se calculado a partir do primeiro dia do diestro determinado pela citologia vaginal (Nelson \& Couto, 2015). Essa dificuldade em se estabelecer a idade da gestação canina, ocorre porque a cadela possui um ciclo reprodutivo com características peculiares como ovulação de oócitos imaturos, longa viabilidade dos espermatozoides, e as diferentes taxas de clivagem embrionária dependentes da maturação oocitária no momento da fecundação (Luz et al., 2005).

Quando o histórico de cobertura não pode ser determinado e a cadela está sendo examinada pela primeira vez para o diagnóstico de gestação, o dia antes do parto pode ser previsto pela ultrassonografia, avaliando o crescimento fetal e a predição é expressa como dias antes do parto em vez de dias de gestação (Luvoni \& Beccaglia, 2006). Além das peculiaridades relacionadas à fisiologia reprodutiva canina, o tempo da gestação parece variar também com a raça do cão e o tamanho da ninhada (Johnson, 2008). Em estudo realizado com 13 cadelas de seis diferentes raças, sendo cinco raças de grande porte e uma de pequeno porte, concluiu que a duração da gestação na raça de pequeno porte foi significativamente maior do que a das raças de grande porte. No mesmo estudo, o tamanho da ninhada foi correlacionado negativamente com a duração da gestação na população total (Okkens et al., 2001).

\section{A ultrassonografia para diagnóstico de gestação canina}

A ultrassonografia é um método diagnóstico de gestação não invasivo e que não oferece risco para a mãe, para os filhotes e para o técnico (Monteiro, 2008). Além disso, o exame ultrassonográfico pré-natal pode avaliar de forma sistemática todas as fases da gestação, monitorando o crescimento fetal por meio da sua biometria anatômica (Freitas \& Silva, 2008). No entanto, a ultrassonografia não é o método de escolha para a avaliação do tamanho da ninhada. O campo de visão restrito criado pelo transdutor e a natureza tortuosa dos cornos uterinos caninos impede uma avaliação contínua dos cornos individualmente (Correia et al., 2012). Existem alguns métodos, descritos pela literatura veterinária, de se utilizar o ultrassom para a realização dos diagnósticos gestacionais em cadelas. São eles: modo A, Doppler e modo B (Kustritz, 2005). A técnica ultrassônica Modo A utiliza como diagnóstico de prenhez a detecção de líquido. Caso a detecção esteja associada a presença de quantidades significativas de 
fluido, este método apresenta como desvantagem a possibilidade de erros de interpretação dos resultados, tanto de prenhez positiva (por exemplo, na hidrometra) quanto de negativa (quando a gestação está no início ou no final, devido à pouca quantidade de líquido na vesícula embrionária/fetal) (Cruz \& Freitas, 2001).

O exame ultrassonográfico em modo Doppler, fornece um sinal audível, que identifica os batimentos cardíacos fetais, diagnosticando a presença de fetos vivos, auxiliando no diagnóstico de sofrimento fetal (Feliciano et al., 2007). O modo de imagem B é um método convencionalmente utilizado em ultrassonografia, permitindo a obtenção de imagens bidimensionais (Pranto, 2009). Permite a avaliação do status gestacional, número de fetos, viabilidade fetal, investigação uterina e estruturas abdominais extra-reprodutivas (Kustritz, 2005). Sendo, portanto, o modo mais utilizado durante o acompanhamento gestacional em cadelas.

Entre os dias 17 a 19, a gestação pode ser diagnosticada, pois ocorre a implantação do blastocisto, no qual é identificado como uma estrutura focal, cranial à bexiga, esférica e anecóica (vesícula embrionária), circundada por uma parede hipoecóica (útero e placenta) (Serra \& Guimarães, 1996). Todavia, clinicamente, recomenda-se realizar o exame ultrassonográfico para o real diagnóstico de gestação em cadelas a partir do $30^{\circ}$ dia após o último acasalamento, pois neste período os sacos gestacionais podem ser identificados com maior precisão e confiança (Farrow, 2006). O embrião pode ser visibilizado primeiramente entre os dias 23 a 25 como uma estrutura ecogênica oblonga de alguns milímetros de comprimento, dentro da membrana fetal esférica (Castro, 2006).

A atividade cardíaca é observada ao mesmo tempo em que o embrião é identificado, do $23^{\circ}$ ao $25^{\circ}$ dia após o pico de LH e o movimento fetal é observado pelo $33^{\circ}$ ao $35^{\circ}$ dia (Kim \& Son, 2007). A atividade cardíaca do feto é de, aproximadamente, o dobro da atividade cardíaca materna. A média dos batimentos cardíacos fetais é de 230 batimentos por minuto (bpm). Com a proximidade do parto, há uma redução desses valores entre 180 a $150 \mathrm{bpm}$ (Zone \& Wanke, 2001). A partir do $30^{\circ}$ dia de gestação inicia-se a organogênese. Nos dias $31^{\circ}$ ao $35^{\circ}$ visibiliza-se no cérebro o plexo coróide ecogênico, circundado por um ventrículo cerebral anecóico (Nyland \& Mattoon, 2005). O esqueleto fetal começa a ser visualizado entre o $33^{\circ}$ e o $39^{\circ}$ dia quando passa a surgir discretas sombras acústicas (Aissi \& Slimani, 2008). A vesícula urinária e o estômago são os primeiros órgãos abdominais a serem visibilizados a partir do $35^{\circ}-39^{\circ}$ dia e aparecem como um foco circunscrito de área anecóica (Castro, 2006). Os pulmões e fígado são inicialmente isoecogênicos, porém entre o $38^{\circ}$ e o $42^{\circ}$ dia, os pulmões tornam-se hiperecóicos em relação ao fígado (Jarreta, 2004). Por fim, tem-se a formação das alças intestinais, vistas somente entre o $57^{\circ}$ e o $63^{\circ}$ dias de gestação (Moriyoshi et al., 1996). Por ser um dos últimos órgãos a se formar, a sua visualização é um dos métodos indicadores de maturação fetal. A sequência dos eventos das características gestacionais para cada fase parece ser similar entre as diferentes raças. Isto porque não há influência da raça na duração da gestação (England, 1998). Por diferentes estágios de desenvolvimento fetal detectáveis pela ultrassonografia, é possível se estimar a idade gestacional. A identificação de diferentes estruturas em datas específicas possibilita a realização deste estudo ultrassonográfico (Carvalho, 2004).

\section{Determinação da idade gestacional por ultrassonografia}

A determinação precisa da idade gestacional por ultrassonografia transabdominal em cadelas é muito importante tanto para se planejar uma cesariana eletiva, tendo a segurança de que a formação fetal está realmente concluída (Kutzler et al., 2003), quanto para fornecer um conforto aos proprietários, garantido uma melhor organização do ambiente para a chegada da ninhada (Silva, 2013). A idade gestacional (IG) e o dia antes do parto (DAP), em cadelas, podem ser estimados através da mensuração de estruturas fetais e extrafetais, que serão escolhidas a depender do período gestacional da fêmea. Essa estimativa é possível, pois a taxa de desenvolvimento embriológico canina está diretamente relacionada com o pico pré-ovulatório de LH, sendo essencialmente útil quando a data da cópula é desconhecida (Jhonson, 2008).

Os parâmetros mais frequentemente utilizados são o comprimento crânio caudal (CCC) (Figura 1), diâmetro biparietal (DBP) (Figura 2), diâmetro abdominal (DAB) (Figura 3) como estruturas fetais, e o interior da cavidade coriônica (ICC) (Figura 4) e a espessura de placenta (EP) (Figura 5) como estruturas extra-fetais (Beccaglia et al., 2016). 


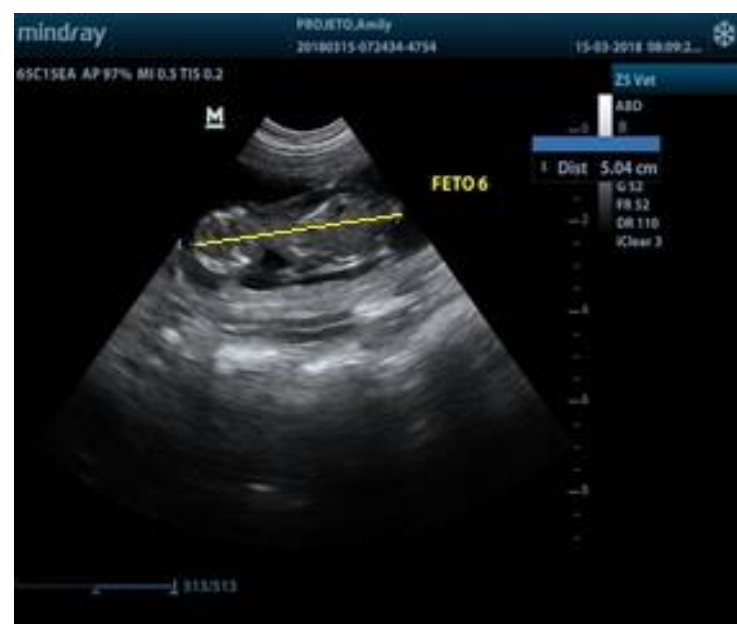

Figura 1. Comprimento crânio caudal (CCC) de um feto da raça Chihuahua, mensurado por ultrassonografia abdominal da cadela gestante

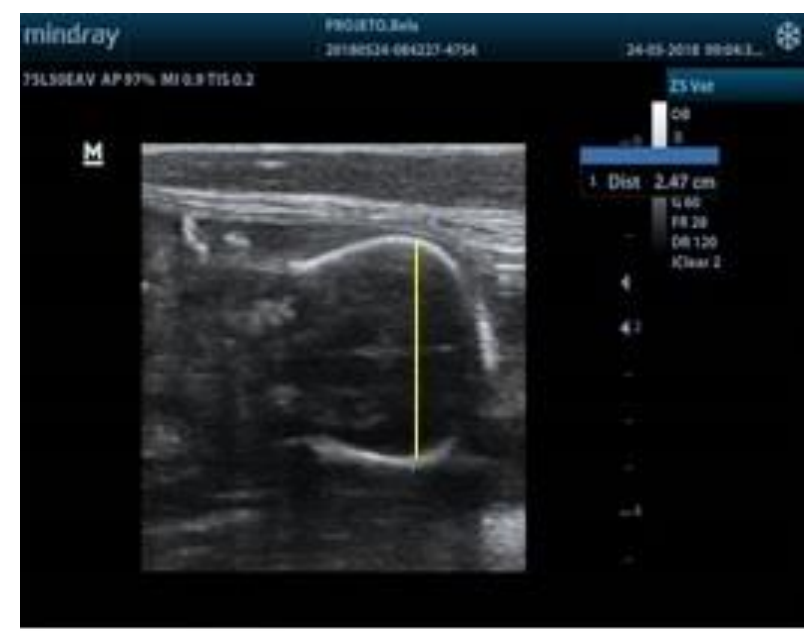

Figura 2. Diâmetro Biparietal (DBP) de um feto da raça Yorshire, mensurado por ultrassonografia abdominal da cadela gestante

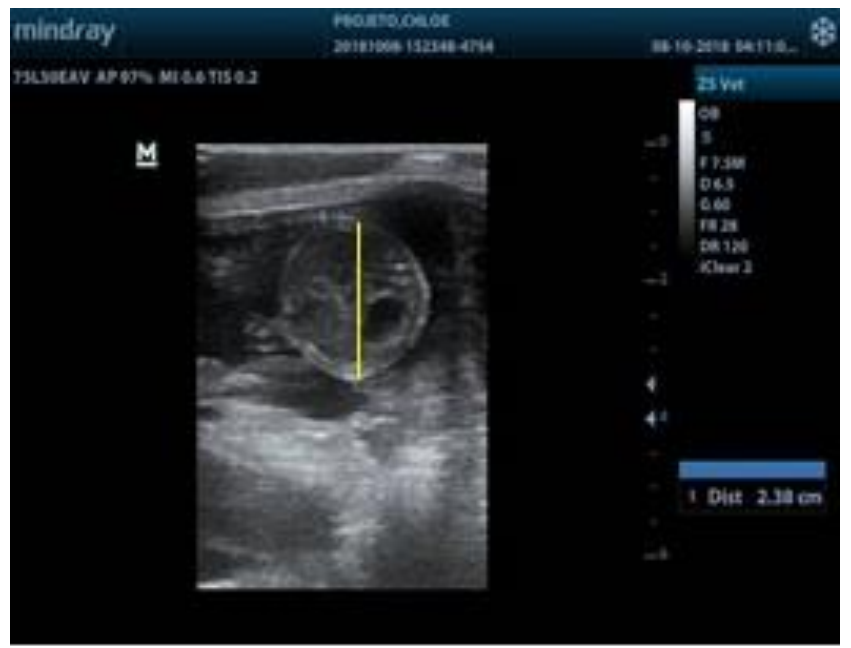

Figura 3. Diâmetro Abdominal (DAB) de um feto da raça Yorshire, mensurado por ultrassonografia abdominal da cadela gestante

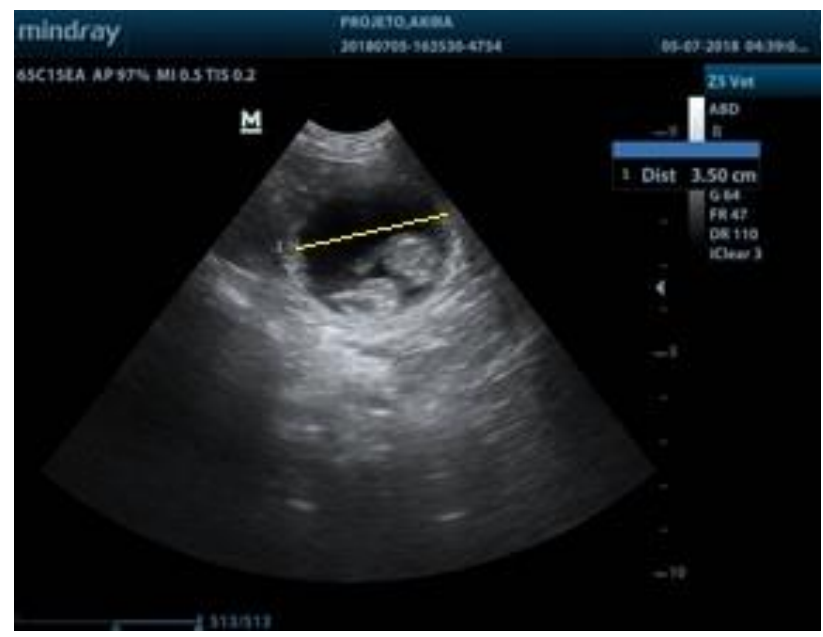

Figura 4. Interior da Cavidade Coriônica (ICC) de um feto da raça Shih-tzu, mensurado por ultrassonografia abdominal da cadela gestante

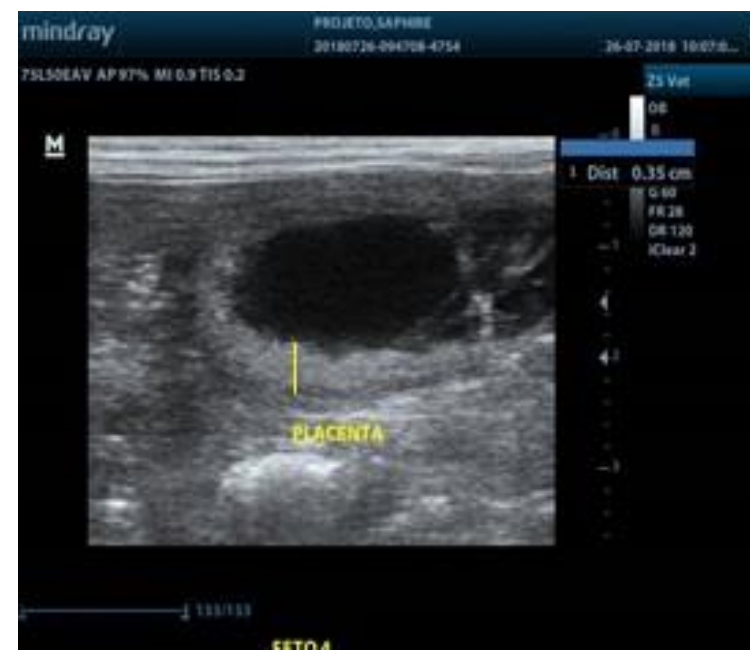

Figura 5. Espessura de Placenta (EP) de um feto da raça Chihuahua, mensurado por ultrassonografia abdominal da cadela gestante 
Ao fazer medições de estruturas fetais ou extra-fetais, pelo menos, dois fetos distintos ou sacos gestacionais devem ser medidos sempre que possível. Em casos de gestação única, a medição de vários parâmetros (ICC, DC, CCC, DBP ou EP) podem ser feitas para aumentar a precisão da estimativa (Luvoni \& Beccaglia, 2006). O diâmetro da vesícula gestacional (cavidade coriônica) é considerado o mais preciso indicador de idade gestacional na cadela entre os dias $20^{\circ}$ e $37^{\circ}$. Porém, pode-se considerar também o comprimento crâniocaudal do feto para se obter esta estimativa de idade nesta mesma fase (Baba, 2010). Contudo, foi observado que é difícil mensurar o comprimento crâniocaudal fetal após $48^{\circ}$ dias pela flexão e pelo tamanho do feto, os quais excedem o campo da imagem do transdutor (Castro, 2006). Já o DBP é um parâmetro confiável no terço final da gestação, entre os dias 38 e 60 (Beccaglia \& Luvoni, 2012).

Sabe-se que o tamanho da cadela pode influenciar a acurácia da previsão do parto, sendo o uso mais preciso nas cadelas de médio porte do que em cadelas pequenas e gigantes (Kutzler et al., 2003). Fato esse demonstrado em estudo, no qual avaliou-se cadelas da raça Rottweiler e Cocker Spaniel Americano, e foi observado diferenças significativas na previsão do parto, podendo levar a erros de até 7 dias a cada centímetro medido, de acordo com a estrutura mensurada (Teixeira et al., 2003).

Em trabalhos realizados o DBP e ICC tiveram uma alta acurácia na previsão do parto em raças de pequeno a médio porte (Beccaglia \& Luvoni, 2006; Kutzler et al., 2003; Luvoni \& Grioni, 2000; Socha \& Janowski, 2014). Em um outro estudo, dessa vez, utilizando raças gigantes, observou-se, também, alta correlação com a previsão do DAP, utilizando a mensuração do DBP e ICC (Alonge et al., 2016). Tadavia, existe trabalho que indica o DBP e DC como medidas de maior correlação com a previsão da data do parto em cadelas de raças gigantes (Teixeira et al., 2009). A precisão máxima da previsão é obtida quando fórmulas para tamanhos específicos (tamanho pequeno e médio) são usadas (Beccaglia et al., 2016), O cálculo da idade gestacional é apenas uma estimativa, pois pode haver erros provenientes da mensuração, visto que há produção de artefatos na imagem, assim como influência de outros fatores dentro de uma mesma raça, como o desenvolvimento corporal e fetal, além do tamanho da ninhada (Jarreta, 2004).

A partir da mensuração, através da ultrassonografia, das estruturas mencionadas, fórmulas foram desenvolvidas e utilizadas na rotina da clínica veterinária (Beccaglia \& Luvoni, 2012):

$$
\begin{aligned}
& \mathrm{DAP}=(\mathrm{ICC} \mathrm{mm}-68.68) / 1.53(\text { para cadelas de pequeno porte }) \\
& \mathrm{DAP}=(\mathrm{ICC} \mathrm{mm}-82.13) / 1.8 \quad(\text { para cadelas de médio porte }) \\
& \mathrm{DAP}=(\mathrm{DBP} \mathrm{mm}-25.11) / 0.61(\text { para cadelas de pequeno porte }) ; \\
& \mathrm{DAP}=(\mathrm{DBP} \mathrm{mm}-29.18) / 0.7(\text { para cadelas de médio porte }) ;
\end{aligned}
$$

No entanto, as variações raciais em cadelas podem interferir nos cálculos da idade gestacional a partir de medidas fetais, sendo necessário estabelecer padrões de medidas e cálculos para animais de um mesmo porte ou característica racial (Melo et al., 2006).

\section{Considerações finais}

A ultrassonografia mostra-se o exame responsável por mostrar aos proprietários informações sobre o desenvolvimento e viabilidade fetal. Pela ultrassonografia é possível realizar a mensuração fetal, no qual visa estimar a idade dos fetos ou data provável do parto. A estimativa da idade gestacional mostrase subjetiva devido a variações relacionadas aos diversos tamanhos das cadelas. Tendo, portanto, a necessidade de se padronizar fórmulas de acordo com essa variação.

\section{Referências bibliográficas}

Aissi, A., \& Slimani, C. (2008). Time of initial detection of fetal structures and anatomic differentiation by using B-mode ultrasound examination in bitches. Pakistan Journal of Biological Sciences, 11(13), $1750-1753$.

Alonge, S., Beccaglia, M., Melandri, M., \& Luvoni, G. C. (2016). Prediction of whelping date in large and giant canine breeds by ultrasonography foetal biometry. Journal of Small Animal Practice, 57(9), 479-483. 
Beccaglia, M., Alonge, S., Trovo', C., \& Luvoni, G. C. (2016). Determination of gestational time and prediction of parturition in dogs and cats: an update. Reproduction in Domestic Animals, 51, 12-17.

Beccaglia, M., \& Luvoni, G. C. (2006). Comparison of the accuracy of two ultrasonographic measurements in predicting the parturition date in the bitch. Journal of Small Animal Practice, 47(11), 670-673.

Beccaglia, M., \& Luvoni, G. C. (2012). Prediction of parturition in dogs and cats: accuracy at different gestational ages. Reproduction in Domestic Animals, 47, 194-196.

Carvalho, C. F. (2004). Ultra-sonografia em pequenos animais. Roca.

Concannon, P., Tsutsui, T., \& Shille, V. (2001). Embryo development, hormonal requirements and maternal responses during canine pregnancy. Journal of Reproduction and Fertility. Supplement, 57, $169-179$.

Concannon, P. W., England, G., III.J., V., \& Linde-Forsberg. (2003). Recent Advaces in Small Animal Reproduction.

Concannon, P. W., McCann, J. P., \& Temple, M. (1989). Biology and endocrinology of ovulation, pregnancy and parturition in the dog. Journal of Reproduction and Fertility. Supplement, 39, 3-25.

Correia, V. J., Feliciano, M. A. R., Melo, D. G., Leite, C. A. L., Neves, C. C., \& Vicente, W. R. R. (2012). Ultrassonografia gestacional em cadelas-revisão de literatura. Nucleus Animalium, 4(2).

Cruz, J. F., \& Freitas, V. J. F. (2001). A ultra-sonografia em tempo real na reprodução de caprinos. Ciência Animal, 11(1), 53-61.

England, G. C. W. (1998). Ultrasonographic assessment of abnormal pregnancy. Veterinary Clinics: Small Animal Practice, 28(4), 849-868.

Farrow, C. S. (2006). Veterinária-Diagnóstico por Imagem do cão e gato. Editora Roca.

Feliciano, M. A. R., Muzzi, L. A. L., Leite, C. A. L., \& Junqueira, M. A. (2007). Ultra-sonografia bidimensional convencional, de alta resolução e tridimensional no acompanhamento da gestação em cadela. Arquivo Brasileiro de Medicina Veterinária e Zootecnia, 59(5), 1333-1337.

Freitas, J. G., \& Silva, A. R. (2008). Diagnóstico de gestação em cadelas. Revista Brasileira de Reprodução Animal, 32(1), 58-66.

Giannico, A. T., Garcia, D. A. A., Gil, E. M. U., Sousa, M. G., \& Froes, T. R. (2016). Assessment of umbilical artery flow and fetal heart rate to predict delivery time in bitches. Theriogenology, 86(7), $1654-1661$.

Jarreta, G. B. (2004). Ultra-sonografia do aparelho reprodutor feminino. Ultrassonografia Em Pequenos Animais, 14, 181-206.

Johnson, C. A. (2008). Pregnancy management in the bitch. Theriogenology, 70(9), 1412-1417.

Kim, B.-S., \& Son, C.-H. (2007). Time of initial detection of fetal and extra-fetal structures by ultrasonographic examination in Miniature Schnauzer bitches. Journal of Veterinary Science, 8(3), 289-293.

Kustritz, M. V. R. (2005). Pregnancy diagnosis and abnormalities of pregnancy in the dog. Theriogenology, 64(3), 755-765.

Kutzler, M. A., Yeager, A. E., Mohammed, H. O., \& Meyers-Wallen, V. N. (2003). Accuracy of canine parturition date prediction using fetal measurements obtained by ultrasonography. Theriogenology, 60(7), 1309-1317.

Lopate, C. (2018). Gestational aging and determination of parturition date in the bitch and queen using ultrasonography and radiography. Veterinary Clinics: Small Animal Practice, 48(4), 617-638.

Luvoni, G. C., \& Beccaglia, M. (2006). The prediction of parturition date in canine pregnancy. Reproduction in Domestic Animals, 41(1), 27-32.

Luvoni, G. C., \& Grioni, A. (2000). Determination of gestational age in medium and small size bitches using ultrasonographic fetal measurements. Journal of Small Animal Practice, 41(7), 292-294.

Luz, M. R., Freitas, P. M. C., \& Pereira, E. Z. (2005). Gestação e parto em cadelas: fisiologia, diagnóstico de gestação e tratamento das distocias. Revista Brasileira de Reprodução Animal, 29(3/4), 142-150. 
Melo, K. C. M., Souza, D. M. B., Teixeira, M. L. B., Amorim, M. J. A. A. L., \& Wischral, A. (2006). Fetometria ultra-sonográfica na previsão da data do parto em cadelas das raças Cocker Spaniel Americano e Chow-Chow. Ciência Veterinária Nos Trópicos, 9(1), 23-30.

Moriyoshi, M., Wagi, K., Nakao, T., \& Kawata, K. (1996). Observation of the growth process of a beagle embryo and fetus by ultrasonography. Journal of Veterinary Medical Science, 58(5), 443445.

Nelson, R. W., \& Couto, C. G. (2015). Medicina interna de pequenos animais (Issue 1). Elsevier Editora.

Nyland, T. G., \& Mattoon, J. S. (2005). Ultra-som diagnóstico em pequenos animais. Roca.

Okkens, A. C., Teunissen, J. M., Van Osch, W., Van Den Brom, W. E., Dieleman, S. J., \& Kooistra, H. S. (2001). Influence of litter size and breed on the duration of gestation in dogs. Journal of Reproduction and Fertility. Supplement, 193-197.

Reynaud, K., Fontbonne, A., Marseloo, N., de Lesegno, C. V., Saint-Dizier, M., \& Chastant-Maillard, S. (2006). In vivo canine oocyte maturation, fertilization and early embryogenesis: A review. Theriogenology, 66(6-7), 1685-1693.

Serra, E. G., \& Guimarães, K. S. (1996). Avaliação ultrasonográfica da gestação na espécie canina. Clínica Veterinária, São Paulo, 1(5), 18-19.

Socha, P., \& Janowski, T. (2014). Predicting the parturition date in bitches of different body weight by ultrasonographic measurements of inner chorionic cavity diameter and biparietal diameter. Reproduction in Domestic Animals, 49(2), 292-296.

Songsasen, N., \& Wildt, D. E. (2007). Oocyte biology and challenges in developing in vitro maturation systems in the domestic dog. Animal Reproduction Science, 98(1-2), 2-22.

Teixeira, M., Berinson, K., Souza, D. M. B., \& Wischral, A. (2003). Relação entre as medidas fetais tomadas por ultrassonografia e o tempo de gestação em cadelas da raça Rottweiler. Revista Brasileira de Reprodução Animal, 27, 2-10.

Teixeira, M. J., Souza, D. M. B., Melo, K. C. M., \& Wischral, Á. (2009). Estimativa da data do parto em cadelas rottweiler através da biometria fetal realizada por ultrassonografia. Ciência Animal Brasileira, 10(3), 853-861.

Tsutsui, T. (1989). Gamete physiology and timing of ovulation and fertilization in dogs. Journal of Reproduction and Fertility. Supplement, 39, 269-275.

Zambelli, D., \& Prati, F. (2006). Ultrasonography for pregnancy diagnosis and evaluation in queens. Theriogenology, 66(1), 135-144.

Zone, M. A., \& Wanke, M. M. (2001). Diagnosis of canine fetal health by ultrasonography. Journal of Reproduction and Fertility. Supplement, 57, 215-219.

Recebido: 19 de janeiro, 2020.

Aprovado: 13 de fevereiro, 2020.

Disponível online: 23 junho, 2020.

Licenciamento: Este artigo é publicado na modalidade Acesso Aberto sob a licença Creative Commons Atribuição 4.0 (CC-BY 4.0), a qual permite uso irrestrito, distribuição, reprodução em qualquer meio, desde que o autor e a fonte sejam devidamente creditados. 